\title{
Classification and Identification of Voltage Sag Sources in Distribution Network with Harmonic
}

\author{
LIU Yajuan ${ }^{\mathrm{a}}$, ZHU Wu ${ }^{\mathrm{b}}$ \\ College of Electronics and Information Engineering, University of Electric Power, Shanghai \\ 200090,China \\ aemail: liuyajuan725@163.com, bemail:zjmzwzsy@126.com
}

Keywords: harmonic, voltage sag source, EMD Energy Entropy, probability neural network(PNN)

\begin{abstract}
A new method to identify voltage sag sources in power distribution network with harmonic is proposed, this method use the theory of EMD to decompose the voltage signals, and obtain a series of IMF. For different voltage sag sources, the IMF's complexities are not the same, so the concept of the EMD energy entropy character is proposed to measure these complexities. The analysis results show that the EMD energy entropy of three-phase voltage for transformer energization is largest; the single phase grounding fault is second; the induction motor starting is minimum, and is close to the normal voltage signals. Finally, combing with PNN to classify and identify voltage sag sources in power distribution network with harmonic, lots of simulation results show that the proposed method in this paper has a high accuracy, and is significantly better than BPNN classifier.
\end{abstract}

\section{Introduction}

As an IEEE definition, the voltage sag or voltage drip is defined as the short duration decrements (between $0.1 \mathrm{pu} \sim 0.9 \mathrm{pu}$ ) in voltage amplitude from one-half to several seconds ${ }^{[1]}$. In recent years, an increasing number of high capacity impact loads and new energy generators in power grids cause voltage sag problems, which have made the electric power departments and customers attach great attention to the power quality problems.

The researches of voltage sag problems mainly include the following several aspects: The harm of voltage sag ${ }^{[2]}$, the analysis and detection of voltage sag ${ }^{[3-4]}$, the classification and identification of voltage sag sources ${ }^{[5-6]}$ and the setting of the location of voltage sag monitor ${ }^{[7-8]}$. The classification and identification of voltage sag sources is helpful to remove the faults timely, reduce economic loss, allocate and manage the responsibility of voltage sag.

Currently, the major researches about classification and identification of voltage sag sources are as followings. The method proposed in [9] is based on the amplitude of voltage sag, whether the voltage jumps and phase voltages are balanced at the termination of voltage sag and. In [10], uses wavelet entropy as the characteristic of voltage sag signals, and is input into PNN to implement automatic identification of voltage sag sources. A combination of HHT and wavelet energy spectrum to classify the voltage sag sources in [11], this method is simply and reliably, has high accuracy and stronger practicability. In [12], by use of the Mam-dani fuzzy reasoning, and then selects good criterion, finally classifies the voltage sag sources.

There are few existing literatures to research the voltage sag sources in distribution network with harmonic, but the great deal of harmonic may make these methods lose efficacy and can't effectively classify and identify the voltage sag sources.

Therefore, based on the above analysis, firstly, the model of distribution network with harmonic is built in this paper, and the characteristics of voltage sag signals are analyzed. Then the concept of the EMD energy entropy is proposed to describe these characteristics, finally, combing with PNN to classify and identify voltage sag sources in power distribution network with harmonic, lots of simulation results show that the proposed method in this paper has a high accuracy. 


\section{Distribution Network Model with Harmonic}

The distribution network model with harmonic is shown in Fig.1. In actual power grid, the even order harmonic components are relatively few, so this paper only considers odd harmonics. The fundamental amplitude and frequency of power supply voltage is $110 \mathrm{kV}, 50 \mathrm{~Hz}$; and also includes $5 \%$ of each 3, 5, and 7 harmonics, $2 \%$ of each 9, 11, and 13 harmonics in Fig.1.

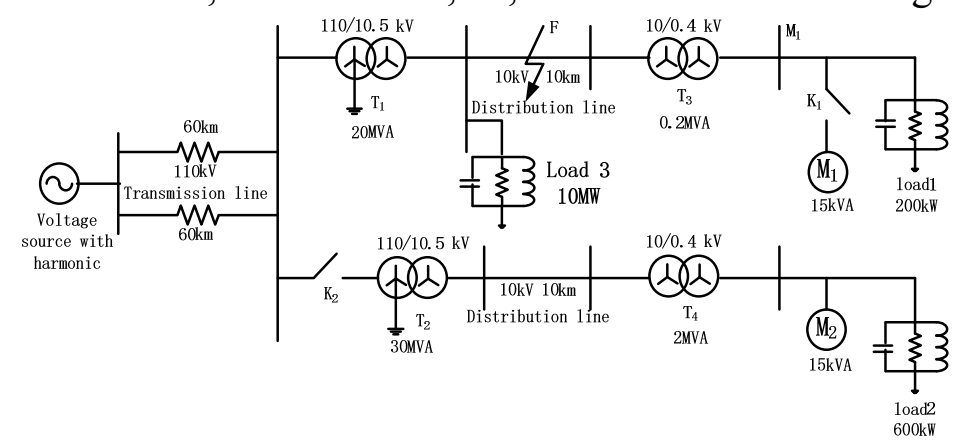

Fig.1 distribution network model with harmonic

Configuration of voltage level is $110 / 10.5 / 0.38 \mathrm{kV} ; \mathrm{F}$ on the distribution line is failure point; M1 is voltage measurement point; K1, K2 is three-phase switch; M (near the load) is induction motor (the capacities of $M_{1}$ and $M_{2}$ are both $15 \mathrm{kV}$ ); the connection mode of transformers $T_{1}$ and $T_{2}$ is $Y n / Y$ type, the $\mathrm{T}_{3}$ and $\mathrm{T}_{4}$ is $\mathrm{Y} / \mathrm{Y}$ type, and $\mathrm{T} 2$ takes the core saturation characteristic into consideration.

\section{The Analysis of Voltage Sag Signals in Distribution Network Model with Harmonic}

Voltage sag sources mainly include short-circuits fault, induction motor starting and transformer energization, etc. The characteristics of voltage sag signals caused by different voltage sag sources are different; the prime problem of accurate identification of voltage sag sources is how to obtain to the characteristics to represent these signals.

(1)Short-circuit fault

In actual power grid operation, the possible short-circuit fault types are: single phase grounding fault, two phase short circuit and three-phase short circuit. Most of the short-circuit faults in distribution network is single phase grounding fault, which is the main reason for voltage sag, so the main research of short-circuit faults is single phase grounding fault. When A-phase ground fault occurs on F point in Fig.1, three-phase voltage sag waveform which is measured on M1 point is shown in Fig.2.

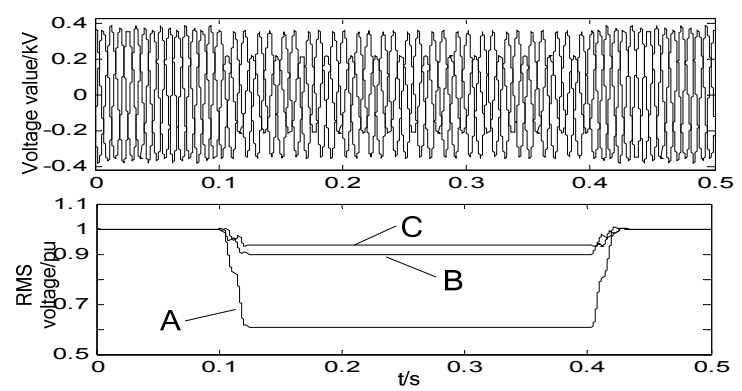

Fig.2 Voltage sag due to single phase grounding fault

By the Fig.2 above, voltage sag signals caused by single phase grounding fault have the following features:

(1)Voltage sag waveforms have a certain degree of distortion;

(2)The degree of voltage sag is serious, the voltage amplitude is lower, and usually less than 0.7 $\mathrm{pu}$;

(3) The voltage amplitude changes in starting and ending time, and keeps unchanged during the voltage sag;

(4) The voltage amplitudes of three phases are not equal. 
(2)Induction motor starting

Currently, induction motors are widely used in industrial manufacturing and daily life, theirs electricity consumption accounts for about $60 \%$ of the total loads in power grid. The starting current of induction motor is larger than rated current four or five times, so it can induce impact on power grid, and then form voltage sag ${ }^{[11]}$. When the induction motor $\mathrm{M}_{1}$ starts, three-phase voltage sag waveform measured on M1 point is shown in Fig.3.

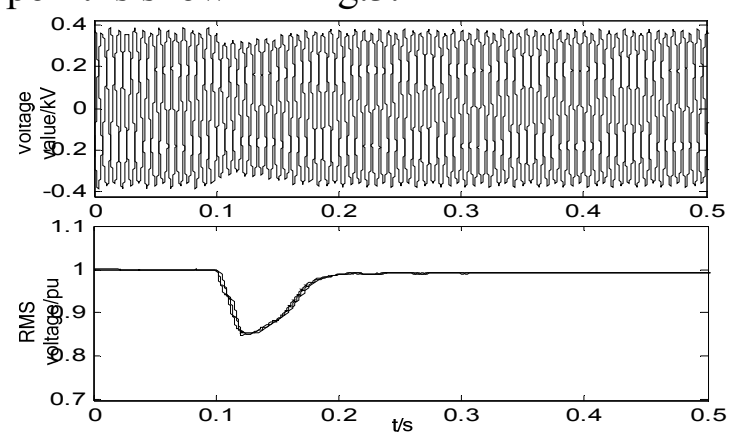

Fig.3 Voltage sag due to induction motor starting

By the Fig. 3 above, voltage sag signals caused by induction motor starting have the following features:

(1)Voltage sag waveforms have a certain degree of distortion;

(2) Voltage sag occurs at the same time and amplitude is consistent;

(3)Voltage sag amplitude is usually greater than or equal to $0.85 \mathrm{pu}$;

(4)The recovery of voltage sag is smooth, no mutations.

(3)Transformer energization

As the result of the core saturation characteristic, the excitation inrush current is $8 \sim 10$ times of rated current in the process of transformer energizing, increases the voltage drop on the system impedance, thus forms voltage sag. When the Transformer $T_{2}$ energizes, three-phase voltage sag waveform measured on M1 point is shown in Fig.4.
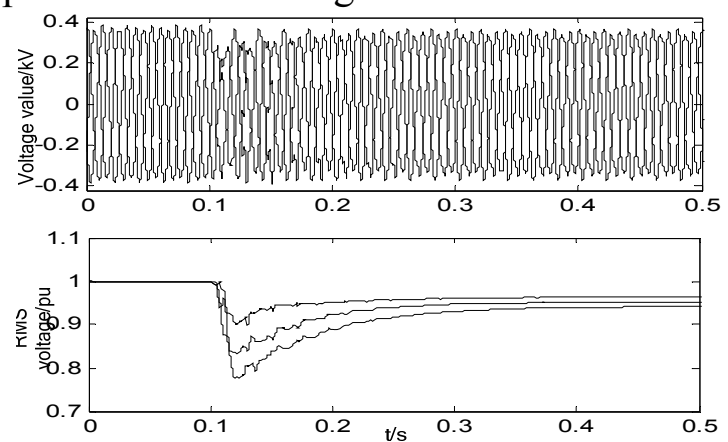

Fig.4 Voltage sag due to transformer energization

By the Fig. 4 above, voltage sag signals caused by transformer energization have the following features:

(1)Voltage sag waveforms have a certain degree of distortion;

(2) Voltage sag amplitude is not consistent, is usually higher than or equal to $0.85 \mathrm{pu}$;

(3)The recovery of voltage sag is gentle, no mutations;

(4) Voltage sag signals have high frequency harmonic in them.

\section{The Method to Classify and Identify Voltage Sag Sources in Distribution Network Model with Harmonic}

The proposed method in this paper to classify and identify voltage sag sources in distribution network with harmonic is based on EMD energy entropy and probability neural network (PNN); the detailed flow chart is shown in Fig.5. 


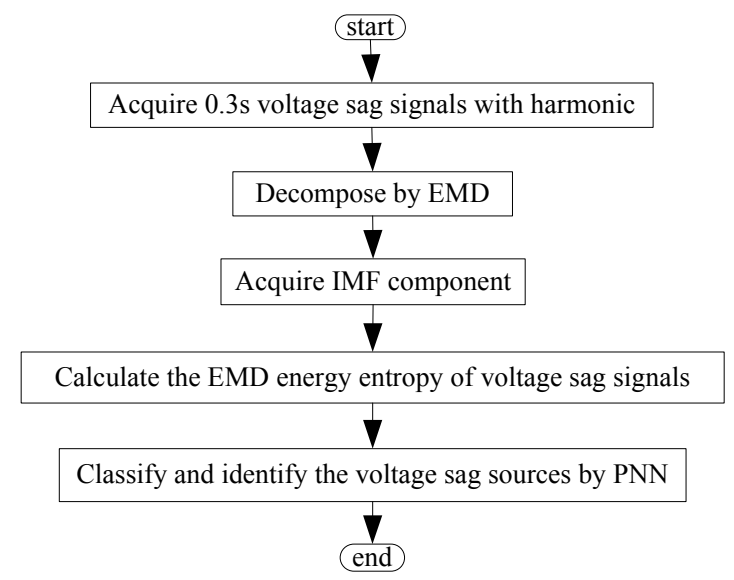

Fig.5 The flow chart of Classification and Identification of Voltage Sag Sources

Firstly, the voltage sag signals are decomposed by EMD, and then a series of IMF is acquired, by use of the IMF to acquire the EMD energy entropy of voltage sag signals, finally, put them into PNN to realize the classification and Identification of voltage sag sources in distribution network with harmonic.

A. Acquire the EMD energy entropy of voltage sag signals

(1) Decompose the voltage sag signals by EMD

Empirical mode decomposition (EMD) decomposes the signals by certain method, and then acquires a series of different frequency scale data sequences, each of them is an IMF (Intrinsic Mode Function). The IMF components must satisfy: (1) at any point, the mean value of the envelope defined by the local maxima and the local minima is zero; (2) the number of extreme and the number of zero crossings must either be equal or differ at most by one [13]. After the decomposition of voltage sag waveforms in Fig. 2 Fig.4, the voltage sag signals meet the two constraints, so the voltage sag signals can be decomposed by EMD.

In addition, EMD absorbs the advantage of wavelet: multiresolution, avoids the problem of wavelet to determine decomposition scale and select wavelet base, is adaptive, thus it can well analyze the nonlinear and non-stationary voltage sag signals ${ }^{[14]}$.

The process of decomposing voltage sag signals by EMD is as following.

(1)Find out all the extreme value points of voltage sag signal $s(t)$, and then respectively get the above and below envelopes of voltage sag signals by Splinefunction; Calculate the average of above and below envelopes, and get the average envelope $m_{1}(t)$, the signal $s(t)$ minus $m_{1}(t)$, finally, get new time series $h_{1}(t)$.

$h_{1}(t)=s(t)-m_{1}(t)$

If $h_{1}(t)$ satisfies the two constraints, it is the first-order IMF component of $s(t)$.

(2) Generally, $h_{1}(t)$ can't be a stable data sequence, So the decomposition steps above are repeated. The process above is repeated $k$ times until the average envelope approaches 0 , get the first IMF component, notes for $c_{1}(t)$, at the same time, $c_{1}(t)$ is the highest frequency component obtained by EMD decomposition.

$$
\begin{aligned}
& h_{1 k}(t)=h_{1(k-1)}-m_{1 k}(t) \\
& h_{1 k}(t)=c_{1}(t)
\end{aligned}
$$

(3) Separate the high frequency component $c_{1}(t)$ from voltage sag signal $s(t)$, and acquire a new time sequence $r_{1}(t)=s(t)-c_{1}(t)$, regard $r_{1}(t)$ as a new time sequence, repeat the above operation, respectively obtain component $c_{1}(t), \quad c_{2}(t), \quad c_{3}(t), \quad c_{4}(t), \ldots c_{\mathrm{n}}(t)$.

$$
\left\{\begin{array}{l}
r_{2}(t)=r_{1}(t)-c_{2}(t) \\
r_{n}(t)=r_{n-1}(t)-c_{n}(t)
\end{array}\right.
$$

When $r_{\mathrm{n}}(t)$ is a monotonic function, decomposition process is terminated, the voltage sag signal $s(t)$ can be described by formula(5):

$$
s(t)=\sum_{i=1}^{n} c_{i}(t)+r_{n}(t)
$$


In the formula above, $c_{1}(t), c_{2}(t), c_{3}(t), c_{4}(t), \ldots c_{\mathrm{n}}(t)$ is a series of IMF decomposed from the voltage sag signal $s(t)$, which represent the frequency components of voltage sag signals in different frequency band, is changing from high frequency to low frequency, accurately highlight the local characteristics of signals; $r_{\mathrm{n}}(t)$ is the residual function, which represents the approximate change trend of voltage sag signals.

(2) Calculate the EMD energy entropy of voltage sag signals

Comparing Fig. 2 Fig.4 can acknowledge, because of different voltage sag sources, the corresponding voltage waveforms are different, so are the IMF. Therefore, it needs some characteristics to describe these complexities of voltage sag signals; herein, the concept of the EMD energy entropy character is proposed ${ }^{[15]}$.

Set $E_{1} 、 E_{2} \ldots E_{\mathrm{n}}$ as the energy of IMF components, which is obtained from EMD decomposition, and $\sum_{i=1}^{n} E_{i}=E$. Set $p_{i}=E_{i} / E$, there is $\sum_{i=1}^{n} p_{i}=1$, the corresponding EMD energy entropy is defined as:

$$
H=-\sum_{i=1}^{n} p_{i} \ln p_{i}
$$

The EMD energy entropy can analyze every IMF component from the perspective of energy, quantify the complexity of each IMF component, and then quantify the complexities of different voltage sag signals, so the EMD energy entropy can be taken to quantify the characteristics of different source voltage sag signals.

B. Classification and identification of voltage sag sources in distribution network with harmonic based on PNN

After extracting the EMD energy entropy of all voltage sag signals, it needs a classifier to identify the voltage sag sources, so probabilistic neural network (PNN) what essence is as classifier is proposed to realize the classification and identification of voltage sag sources.

The characteristics of probabilistic neural network (PNN) are: fixed model, high training speed and can output the Bayes posterior probability, etc. It is a forward neural network, has a total of four layers, and its structure is shown in Fig.6.

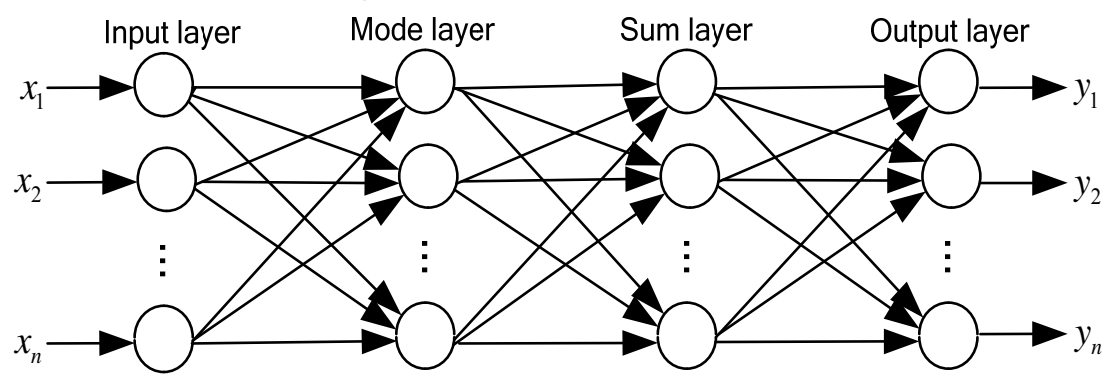

Fig.6 The structure of probability neural network

(1) Input layer

Input layer receives the EMD energy entropy vector $x$ of voltage sag signals, passes it to the model layer by linear transfer function; the number of neurons is in accordance with the dimensions of the vector $x$, and the input and output of each neuron and are single.

(2)Mode layer

The model of training samples in mode layer is corresponding to the EMD energy entropy vector $x$, the number of neurons is equal to the total number of training samples of voltage sag signals, the output of each neuron is:

$$
f\left(x, w_{i}\right)=\exp \left[-\frac{\left(x-w_{i}\right)^{T}\left(x-w_{i}\right)}{2 \sigma^{2}}\right](i=1,2, \mathrm{~L} n)
$$

In the formula above: $w_{i}$ is the connection weight between input layer and model layer; $\sigma$ is smoothing parameter.

(3)Sum layer

In sum layer, all the probabilities of certain voltage sag are added and multiplied by the price factor, then obtain the estimate probability density function of various voltage sag sources. The 
number of neurons in this layer is equal to the types of voltage sag sources, the types are corresponding to the units in sum layer, the units in sum layer only sum with the same type units in model layer, have no connection with other units.

(4)Output layer

The output layer mainly performs the function of judgment and classifies the voltage sag sources, the neurons are also called competition neurons, which are corresponding to the types of voltage sag sources; the number of neurons are corresponding to types of voltage sag sources.

Therefore, the extracted EMD energy spectrum entropy of voltage sag signals is input into probability neural network and then can well realize the classification and recognition of voltage sag sources in distribution network with harmonic.

\section{Simulation and Analysis}

A. The extraction and analysis of the EMD energy entropy of voltage sag signals

According to the simulation model in Fig.1, respectively do the simulations as followings.

1) For the voltage sag caused by single phase grounding fault, 100 group's data of voltage sag are measured respectively under the conditions of different loads, different fault locations, different sag duration and different transition resistance.

2) For the voltage sag caused by induction motor starting, 100 group's data of voltage sag are measured respectively under the conditions of different sag duration, different line loads and different capacities of induction motor.

3) For the voltage sag caused by transformer energization, 100 group's data of voltage sag are measured respectively under the conditions of different loads, different switched time and different capacities of transformer.

4) For the normal conditions, 50 group's data of voltage sag are measured respectively under the conditions of different loads and different capacities of transformer.

The EMD energy entropy of the data in every group is calculated, which is shown in Fig.7.
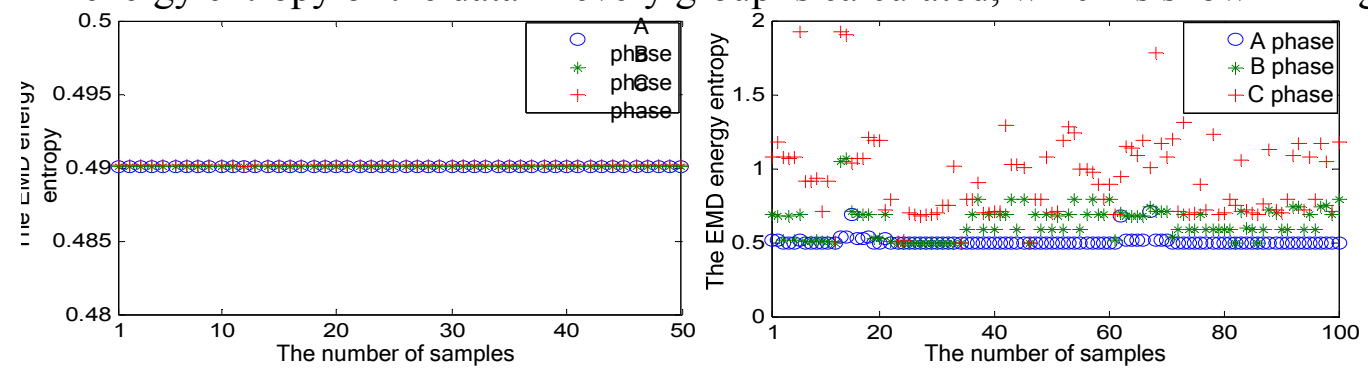

(a) The normal voltage

(b) Single phase grounding fault
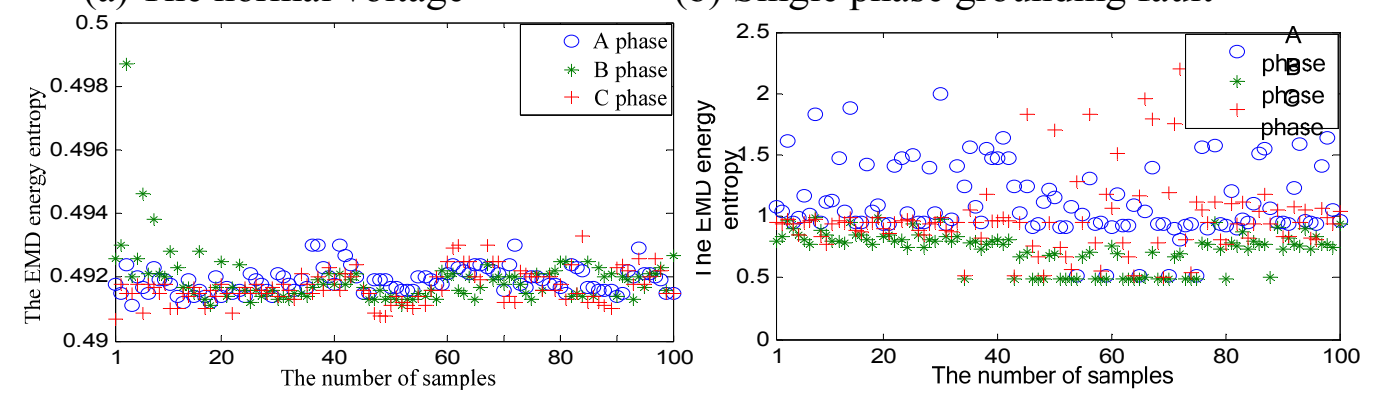

(c) Induction motor starting

(d) Transformer energization

Fig.7 the EMD energy entropy of voltage signals

By the Fig.7 above, the EMD energy entropy of voltage sag signals is bigger than normal voltage signals, this is because other than the harmonics considered in distribution network, there are also other high frequency components during the voltage sag, thus makes the voltage sag signals' uncertainty higher, and then makes the EMD energy entropy bigger. The detailed analysis is as following.

(1) The voltage sag caused by transformer energization: The three-phase's EMD energy entropy of this kind is bigger than the other two kinds'. 
(2) The voltage sag caused by induction motor starting: The three phase voltages' EMD energy entropy of this kind is close to the normal voltages', and each phase voltage's EMD energy entropy is smaller than the other two kinds'.

(3) The voltage sag caused by single phase grounding fault: each phase voltage's EMD energy entropy of this kind is between the other two kinds'. The B phase and C phase's EMD energy entropy of this kind is bigger, but the A phase and C phase's EMD energy entropy of the voltage sag caused by transformer energization is bigger.

Therefore, the EMD energy entropy can be used to distinguish the three kinds of voltage sag sources, and can be used to classify and identify the voltage sag sources in distribution network with harmonic.

B. The classification and identification of voltage sag sources based on PNN

PNN can be directly created by the function: net = newpnn $(\mathrm{P}, \mathrm{T}$, and $\mathrm{S})$, which is provided by Matlab; in the function, $\mathrm{P}$ is the input vector (The EMD energy entropy of voltage sag signals), $\mathrm{T}$ is the target vector, $\mathrm{S}$ is the extension parameter. In this paper, the types of voltage sag sources are the output of PNN. The BPNN is used to compare with PNN in this paper; the BPNN's nodes of input layer and output layer are the same as PNN's.

For the voltage sag signals, 150 group's data are obtained from the three types of voltage sag sources, each type has 50 group's data. All the data are used to expand training the PNN (the parameter $\mathrm{S}$ is 0.01 ), after the completion of training, the remaining 150 group's data are input into PNN for testing; the same data are input into BPNN to train and test, the comparison results are shown in Tab.1.

Tab.1 Comparison of training test results

\begin{tabular}{ccccc}
\hline categories & $\begin{array}{c}\text { Samples in } \\
\text { training set }\end{array}$ & $\begin{array}{c}\text { Samples in test } \\
\text { set }\end{array}$ & \multicolumn{2}{c}{ Correct recognition rate } \\
\hline $\begin{array}{c}\text { Single phase } \\
\text { grounding fault } \\
\text { induction }\end{array}$ & 50 & 50 & PNN & BPNN \\
$\begin{array}{c}\text { motor starting } \\
\text { transformer } \\
\text { energization }\end{array}$ & 50 & 50 & $88 \%$ & $92 \%$ \\
\hline
\end{tabular}

From the test results above, the correct recognition rate of PNN is significantly higher than BPNN; The EMD energy entropy can well represents the characteristics of voltage sag signals in distribution network with harmonic, a combination of EMD energy entropy and PNN can well classify and identify the voltage sag sources.

\section{Conclusions}

For the classification and identification of voltage sag sources in distribution network with harmonic, the characteristics of three types of sag sources are analyzed, and the concept of the EMD energy entropy is proposed to describe these characteristics, finally, combining with PNN to classify and identify the voltage sag sources. The main conclusions are as followings:

1) The three-phase's EMD energy entropy of voltage sag source caused by transformer energizing is maximum; the single-phase grounding fault's is second; the induction motor starting's is close to the normal voltage's, and is smaller than the other two kinds'.

2) The three-phase's EMD energy entropy of three voltage sag sources has distinct characteristics, three voltage sag sources can be classified and identified by this.

3) The correct recognition rate of PNN is significantly higher than the BPNN's.

4) The correct recognition rate of voltage sag sources in distribution network with harmonic based on the combination of the EMD energy entropy and PNN is higher than $88 \%$, and the proposed method in this paper can well classify and identify the voltage sag sources in distribution network with harmonic. 


\section{Acknowledgement}

In this paper, the research was sponsored by Shanghai municipal education commission key projects of scientific research and innovation (11ZZ173); Local colleges and universities of Shanghai science and technology innovation action plan ability construction projects (10110502200,11510500900).

\section{References}

[1]ZHENG Li, ZHANG Yao, LIN Lingxue. Simulation Research on the Voltage Sag of Distribution Network with Distributed Generations [J]. Southern Power System Technology, 2013, 7(2):64-67.

[2]LIU Xuna, XIAO Xianyong, WANG Ying. Voltage Sag Severity and Its Measure and Uncertainty Evaluation [J]. Proceedings of the CSEE, 2014,34(4) : 644-658.

[3]WANG Dongxu, LE Jian, LIU Kaipei, et al. Voltage Dip Analysis for Multiple Faults Case in Complex Power Grid [J]. Proceedings of the CSEE, 2012,32(7) : 101-106.

[4]Qu Shuo, Huang Chun, Jiang Yaqun, et al. A New Detection Method of Voltage Sag Applied in DVR [J]. Trans of China Electrotechinal Society, 2013, 28(4) : 234-239.

[5]LIU Yingying, WANG Tongxun, FENG Dandan, et al. Multiple Criterions Based Voltage Sag Location Method [J]. Proceedings of the CSEE, 2015,35(1) : 103-111.

[6]ZHOU Chao, TIAN Lijun, HOU Yanwen, et al. Fault Lo-cation Estimation Based on Optimal Voltage Sag Monitori-ng Program [J]. Automation of Electric Power System, 2012,36(16) :102-107.

[7]Zhou Chao, Tian Lijun. An Optimum Allocation Method of Voltage Sag Monitoring Nodes Based on Particle Swarm Optimization Algorithm [J]. Trans of China Electrotechinal Society, 2014,29(4) : 181-187.

[8]CHEN Lipin, XIAO Xianyong, ZHANG Wenhai. Optimalallocation of voltage-sag monitors considering disturbance-source locating [J]. Electric Power Automation Equipment, 2014,34(2) : 79-90.

[9]DING Ning, CAI Wei, SUO Juan, et al. Research on V-oltage Sag Sources Recognition Method[J]. Power SystemTechnology, 2008,32(2) : 55-59.

[10]JIA Yong, HE Zheng-you , ZHAO Jing. A Method to Identify Voltage Sag Sources in Distribution Network Based on Wavelet Entropy and Probability Neural Network [J]. Power System Technology, 2009,33 ( 16 ): 63-69.

[11]QI Bo, ZOU Jin-hui, FAN Yu-gang, et al. Identification of Voltage Sag Source Based on Hilbert-Huang Transformand Wavelet Packet Energy Spectrum [J]. Electric Power, 2013,46(8) : 112-117.

[12]LI Guo-dong, DING Ning, XU Yong-hai. Voltage sag di-sturbance recognition based on Mamdani fuzzy inference[J].Journal of North China Electric Power University, 2010,37(2) : 43-48.

[13]Huang N E , Shen Z ,Long S R . The empirical mode decomposition and the Hilbert spectrum for nonlinear and nonstationary time series analysis[J] . Proceedings of The Royal Society Soc Lond , 1998 , 454(1971) : 903-995 .

[14]YE Lin, LIU Peng. Combined Model Based on EMD-SVM for Short-term Wind Power Prediction [J]. Proceedings of the CSEE, 2011,31(31) : 102-108.

[15]WANG Qingliang, FU Zhouxing. An Adaptive Method for Single-phase-to-ground Fault Line Selection Based on Energy Entropy Measure [J]. Automation of Electric Power System, 2012,36(5) : 103-107. 